\title{
Dynamic Response Measurement of Clinical Gas Analysers
}

\author{
Kabita Shakya ${ }^{1}$, Catherine Deegan ${ }^{1}$, Fran Hegarty ${ }^{2}$, Charles Markham ${ }^{3}$ \\ ${ }^{1}$ School of Informatics and Engineering, Institute of Technology Blanchardstown \\ ${ }^{2}$ Department of Medical Physics and BioEngineering, St. James's Hospital, Dublin 2 \\ ${ }^{3}$ Department of Computer Science, National University of Ireland, Maynooth \\ Kabita.Shakya@itb.ie; Catherine.Deegan@itb.ie; \\ Fhegarty@stjames.ie;Cmarkham@cs.may.ie
}

\begin{abstract}
Capnographs are devices that use $\mathrm{CO}_{2}$ from a patients' expired breath to monitor the cardiopulmonary status during anaesthesia and mechanical ventilation. The waveforms are called capnograms. Capnographs at present are routinely calibrated via a static calibration method only. There is no specific method of dynamically testing capnographs. Since capnograms are time varying, there is a definite requirement to evaluate the dynamic response of these systems.

This paper presents a technique to determine the time and frequency response of capnographs using a custom built EtCO $\mathrm{C}_{2}$ simulator system. This system is computer controlled via a $C$ program. A portable version of the simulator system has also been developed using a microprocessor. The waveforms obtained using the simulator system are recorded and image analysis is performed using Matlab to obtain the time and frequency responses of the capnographs. This paper explains the image analysis part of the technique in detail.

Measurements of several types of capnograph systems proved that older capnographs usually do not satisfy the manufacturer quoted specifications for time and frequency response. Monitoring capnographs on a routine basis is thus important and is recommended.

This new technique of time and frequency response measurement can be used as part of a quality control protocol for capnographs; for commissioning and routine monitoring as well as to verify manufacturer quoted specifications in new units.
\end{abstract}

\section{Introduction}

Monitoring the concentration of exhaled $\mathrm{CO}_{2}$ gas enables monitoring of physiological condition of patients during anaesthesia and mechanical ventilation. Normally, the $\mathrm{CO}_{2}$ concentration of expired breath changes from $0 \%$ to $5 \%$ at the end of expiration. Capnography is the technique that plots $\mathrm{CO}_{2}$ concentration against time. Capnographs are devices that produce such traces (capnograms). These devices also calculate an index referred to as the End Tidal $\mathrm{CO}_{2}$ or $\mathrm{EtCO}_{2}$ which is the maximum concentration of $\mathrm{CO}_{2}$ at the end of the exhaled breath. This value is taken as an indicator of alveolar $\mathrm{CO}_{2}$ concentration at the end of gas exchange and by extension the arterial blood $\mathrm{CO}_{2}$. Capnograms have a standard pattern in a healthy human being and any deviation from this pattern indicates the deterioration in patient condition [1]. To measure the $\mathrm{CO}_{2}$ concentration accurately, the $\mathrm{CO}_{2}$ analyser must respond rapidly[3]. 
This paper presents a new practical method to determine the dynamic behavior of capnographs using a purposely-designed $\mathrm{EtCO}_{2}$ simulator system. This paper is primarily focused on the (image) data acquisition and data analysis parts of the newly developed technique.

\subsection{Originality}

The only method currently used to check the performance of the capnographs is a static calibration method [1]. There is no definitive method available that would facilitate rapid assessment of the dynamic integrity and efficacy of a capnograph system. Since $\mathrm{CO}_{2}$ gas waveforms are time varying, there is a definite requirement to evaluate the dynamic response of these systems. It is intended to address this shortfall using this new technique to:

1) $\mathrm{Be}$ incorporated into the quality control (QC) procedure and /or be used in the construction of a QC protocol for the evaluation of capnograph systems during the routine monitoring and during commissioning of capnographs.

2) Be utilized as a training /diagnostic system in the clinical engineering environment.

Figure 3 shows a plot of a typical capnogram. This plot is the replica of waveform obtained from the $\mathrm{EtCO}_{2}$ analyser of a sidestream capnograph (a type of capnograph where gas analysis occurs away from the sampling site). The total response time, $t_{\text {response, }}$, is the total of $t_{1}$ (transit time or transport delay time) and $t_{2}$ (rise time or sensor response time). Transit time is the time taken for the sample gas to move from the point of sampling to the point of measurement while rise time is the time taken for the analyser output to respond to a sudden step change in carbon dioxide concentration - the time it takes for the analyser output to change from $10 \%$ of the final value to $70 \%$ of the final value.

Rise time can also be defined as the time taken to change the analyser output from 10 to 90 $\%$ of the final value $\left(t_{2}=t{ }_{90}\right)$. The $t_{70}$ value is generally used instead of $t_{90}$ because the $70 \%$ point is on a steeper part of the response curve and therefore less susceptible to noise. For all practical purposes, $t_{90}$ is twice the value of $t_{70}[3]-[9]$.

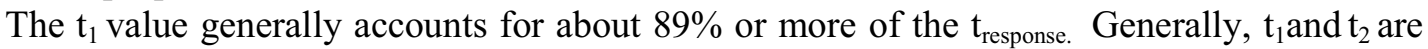
not defined in manufacturers' specifications but these factors should be defined and specified. This is because if $t_{1}$ is long, it can prolong $t_{2}$ (by subjecting the bolus of gas to dispersal caused by convection and diffusion during transit down the catheter/tube); this can affect the waveform morphology and result in underestimation of $\mathrm{EtCO}_{2}$ during high frequency ventilation. In addition, a long $t_{1}$ may introduce an unacceptable delay in the total response time of the analyser [4]. Some findings also suggest that the transit time is more important in attaining a true $\mathrm{EtCO}_{2}$ level [8].

\section{Methods}

For dynamic and frequency response measurements, an $\mathrm{EtCO}_{2}$ simulator system has been developed so that waveforms of desired frequency can be created. The waveform introduced is a step waveform.

\subsection{Description of the $\mathrm{EtCO}_{2}$ simulator system}

The $\mathrm{EtCO}_{2}$ simulator system [Figure 1] simulates the human respiration cycle. It is constructed using $5 \% \mathrm{CO}_{2}$ gas and room air as the gas sources that can be switched alternatively. Both supplies are pressure regulated and connected through non-return valves to 
electric valves. The valves are fast acting valves with a response time of $3 \mathrm{msec}$. These are interfaced to a computer using a DAS-08 card and controlled using a C program. The on/off time of valve is user defined allowing a wide range of waveforms to be simulated. The output from the valves is delivered to a ' $\mathrm{Y}$ ' connector and finally the output from the system is delivered to capnograph [10]. A portable version of the simulator was also developed using a microprocessor (PIC16C57) so that it can be used in clinical environments. This system also uses a small LED circuit to indicate the opening of each valve. The design criteria for the portable system are to ease the application of the system using a keypad configuration and to allow for static calibration as well [11].

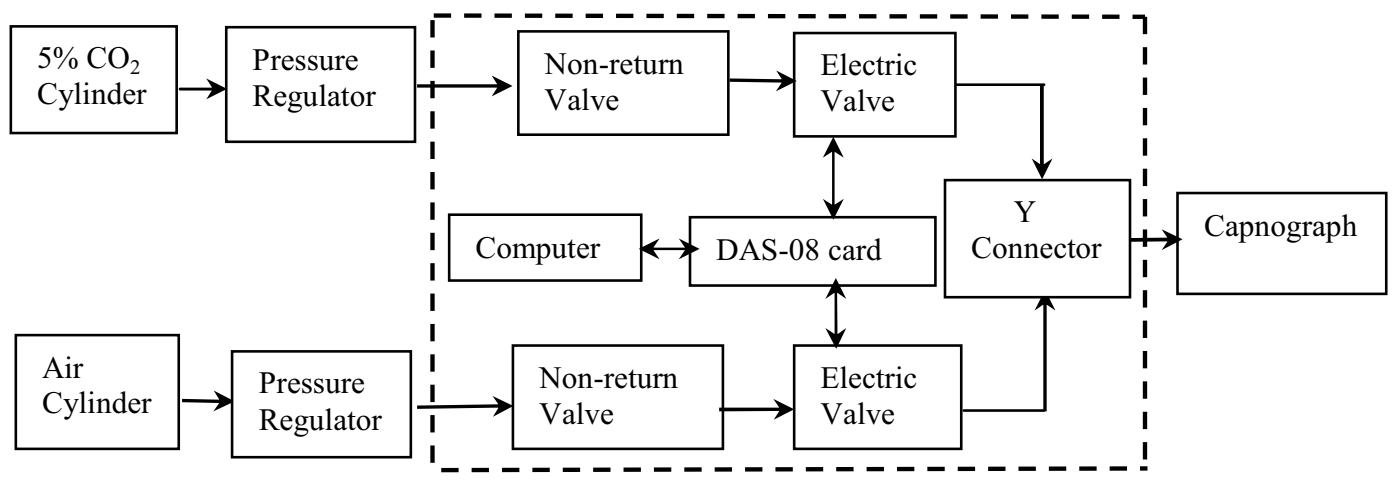

Figure 1. Block diagram of the $\mathrm{EtCO}_{2}$ simulator system

\subsection{Data acquisition and analysis}

For data acquisition, the capnograms along with the LED states are captured by digital video recording (because most capnographs are standalone units and do not give the user easy access to the raw data). The digital video camera recorder used is a Sony DCR-PC8E with a framerate of $25 \mathrm{fps}$ (frames per second). The image data thus recorded is converted to an uncompressed avi file format using a FireWire card (IEEE1394) and a software (VideoWave5). The '.avi' file is then converted into individual frames (.bmp files) using another software (AVI Quick). These frames are then processed using a program in Matlab so that an exact replica of the original capnogram is obtained in a digital format. An image-processing program described briefly below is designed for bright waveforms on black/dark backgrounds.

The matlab program takes two frames to process at one iteration, so as to obtain a digital value of $\mathrm{CO}_{2}$ magnitude that corresponds to a pixel in the real capnogram.

The former frame is subtracted from the latter. The resultant frame is cropped such that the waveform of interest is captured and the data is finally converted to black and white pixel values. The elements of each column of this matrix are added and the resultant column matrix is stored as a vector ' $\mathrm{BW}$ '. This vector will have numerical values in the row; the elements of which had white pixel as their elements and zero (0) values in the row with no white pixel.

The upper leftmost point of cropping (Xmin, Ymin), is considered as the reference point $\mathrm{O}$ $(0,0)$ and other points are measured with reference to that point. The lower rightmost point of cropping is (Xmax, Ymax) [Figure 2]. These values are determined from a bmp image and provided to the program before running the program. Using the 'linspace' command in matlab, 'Ymax-Ymin +1 ' number of equidistant points (in row vector form) are found between Ymin and Ymax, that when transposed and multiplied (array multiplication) with the vector ' $\mathrm{BW}$ ' (which is also of the same order), gives a column vector (with zero and numerical value 
elements). Adding it rowwise gives a value say 'A'. The BW matrix when added rowwise gives a value say ' $B$ '. The value ' $A$ ' when is divided by ' $B$ ' will give the approx. location of the pixel (position of pixel value) that is desired. This single value is then stored as the position value for that iteration.

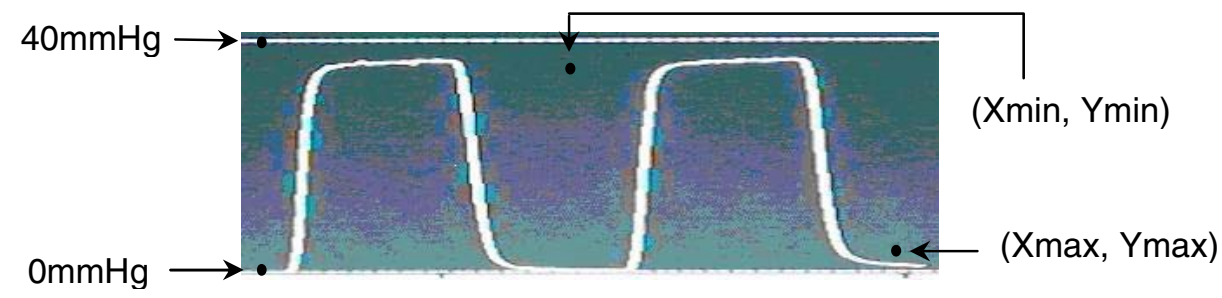

Figure 2. A bmp file of a capnogram trace from Datex Ohmeda, showing the $0 \mathrm{mmHg}$ and 40mmg lines, the cropping points, (Xmin, Ymin) and (Xmax, Ymax)

This pixel position ( $\mathrm{x}$ in equation 1) is then used to calculate the respective $\mathrm{CO}_{2}$ magnitude value (in $\mathrm{mmHg}$ ) using the straight-line equation,

$$
\mathrm{Y}=\mathrm{mx}+\mathrm{C} \text {; Where } \mathrm{m}=\left(\mathrm{Y}_{2}-\mathrm{Y}_{1}\right) /\left(\mathrm{X}_{2}-\mathrm{X}_{1}\right) \ldots \ldots \ldots \ldots \ldots \ldots \ldots \text { Equation } 1
$$

Equation 1 here is used for a plane where the $\mathrm{Y}$-axis has $\mathrm{CO}_{2}$ magnitude values and the $\mathrm{X}$ axis has the pixel position values. Two required points $(\mathrm{ph}, 40)$ and $(\mathrm{pl}, 0)$ are known, where, $\mathrm{ph}$ and $\mathrm{pl}$ are the pixel positions in cropped image of $40 \mathrm{mmHg}$ and $0 \mathrm{mmHg}$ lines. This process is repeated for all iterations. The separation between frames is taken as 2 so that there is a detectable change between the frames. Therefore, the first frame will be subtracted from the third one, the second from the fourth and so on.

The final result is then a series of points (referred here as the ' $\mathrm{CO}_{2}$ magnitude vector') that recreates the capnogram plot, if plotted versus the frametime. The frametime value is found out as iteration number divided by frame rate $(0.04)$ and stored as the 'frametime' vector. The LED states captured enables tracing out when the $\mathrm{CO}_{2}$ valve is open. The 'ON' and 'OFF' state of LED implies the valve 'opening' and 'closing' respectively. The LED state for each iteration is recorded as 'ledstate' vector.

Finally, the program plots the calibrated $\mathrm{CO}_{2}$ magnitude value $\left(\mathrm{CO}_{2}\right.$ magnitude vector $)$ and the LED state (ledstate vector) in the vertical direction versus frame time (the frametime vector) in the horizontal direction that enables the calculation of transit time and time response. A typical capnogram plot is shown in Figure 2 and the capnogram plotted after image analysis is shown in Figure 3.

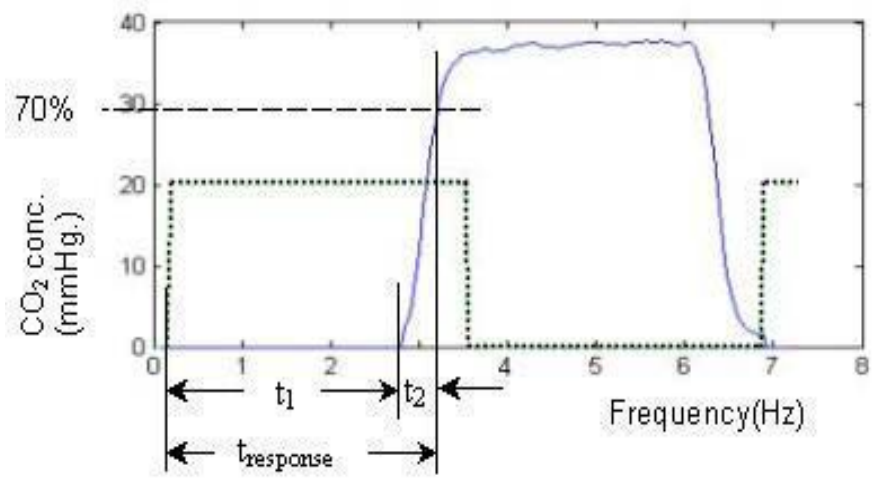

Figure 3. Digital plot labeled showing the transit time $\left(t_{1}\right)$, rise time $\left(t_{2}\right)$ and the total response time $\left(\mathrm{t}_{\text {response }}\right)$ of the capnograph. 
Transit time $\left(\mathrm{t}_{1}\right)$ is calculated as the time difference between when the LED turns ON and the waveform appears. The rise time $\left(\mathrm{t}_{2}\right)$ is calculated as the time it takes for the waveform to change from $0 \%$ to $70 \%$ of the final value. The values $t_{1}, t_{2}$ and $t_{\text {response }}$ are shown in Figure 3.

For determining the frequency response, the maximum value of $\mathrm{CO}_{2}\left(\mathrm{EtCO}_{2}\right)$ is the amplitude value and the waveform frequency (simulated breaths per minute) is the frequency value $[10][11]$.

\section{Results}

The experiments performed using the new technique determined the time and frequency responses of several different capnograph systems [10]-[12].

The frequency response curves for the Drager Capnolog (decommissioned and approximately 15 years old unit) and the Datex Ohmeda, M-CAiO (new) units are presented in Figure 4. This plot shows that the frequency response of the Drager Capnolog is 7.5-60BPM (manufacturer specification: 2-99BPM) [13][14] while that for the Datex Ohmeda is 7.5- 80 BPM (manufacturer specification: 4-80BPM)[16]. The dynamic response obtained also is out of manufacturer specification for the Drager Capnolog while is in agreement for the Datex Ohmeda. The complete set of experiments performed and the results obtained is presented in [12].

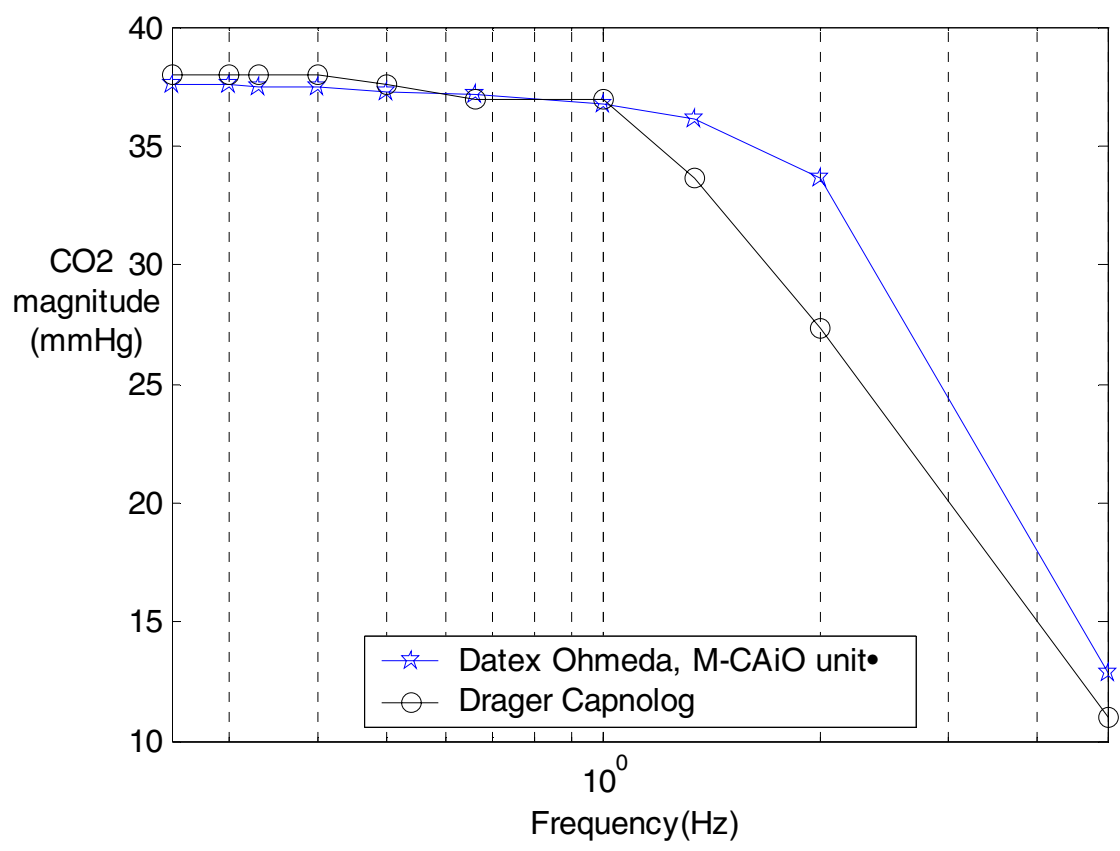

Figure 4. Frequency Response curves for Datex Ohmeda and Drager capnolog

\section{Discussion}

The image processing technique described in this paper allows the time and frequency response measurement for any capnograph type, irrespective of whether the capnograph has a digital outlet for time and frequency response measurements. This technique also avoids the problem of having to interface with several units for the measurement. This method however is time consuming and requires a large amount of memory space to store and process data. 
Further, the frame rate of the camera limits the frequency range. The maximum frequency that can be measured in this experiment is $12.5 \mathrm{~Hz}$ (because of the Nyquist criteria) as the camera captures at $25 \mathrm{fps}$. The minimum frequency depends upon the capnograph screen. Generally, the width of the screen is around $4000 \mathrm{msec}$; this corresponds to a minimum measurable frequency of $0.25 \mathrm{~Hz}$.

\section{Conclusions}

The results verified that the old capnograph units do show deterioration in performance with their age. It is thus recommended that the time and frequency response should be monitored on a regular basis to ensure safe operation [12]. This new technique is developed to resolve this issue. It can be used as a quality control procedure during the routine monitoring or the commissioning of new capnographs or in the construction of the QC protocol for the evaluation of capnograph systems in the clinical environment. It can also be used to ease the purchase decisions of capnographs in hospitals or to verify the manufacturer quoted specifications.

The time and frequency response determination of capnographs is important as it ensures that the capnograms are free of mechanical error and can be interpreted accordingly. Also, it determines if the capnographs can be used in high frequency ventilation schemes.

\section{References}

[1] Kodali. B. S.: Capnography.com [Online], Available: http://www.capnography.com

[2] Guyton A.C., "Textbook of Medical Physiology", $10^{\text {th }}$ Edition, W. B. Saunders Co. Philadelphia, PA.

[3] Brenner J. X and Westenskow D. R., "How the rise time of Carbon Dioxide analysers influences the accuracy of carbon dioxide measurements", Br. J. Anaesth. 1988; 61: 628-638

[4] Breen Peter H., Mazumdar B. and Skinner Sean C., "Capnometer transport Delay: Measurement and Clinical Implications", Anesth Analg 1994; 78: 584 -6

[5] Fletcher R., Werner O., Nordstrom L. and Jonson B., "Sources of error and their correction in the measurement of Carbon dioxide elimination using the Siemens- Elema $\mathrm{CO}_{2}$ analyzer", Br. J. Anaesth. $1983 ; 55: 177-85$

[6] From RP and Scamman FL, "Ventilatory Frequency influences accuracy of End tidal $\mathrm{CO}_{2}$ measurements: analysis of seven capnometers", Anesth Analg 1988; 67: 884-6.

[7] Pascucci RC, Achena JA and Thompson JE, "Comparison of a sidestream and mainstream capnometer in infants", Crit care Med. 1989; 17(6): 560 - 2 .

[8] Schena J, Thompson and Crone RK, " Mechanical influences on the capnogram”, Crit Care Med 1984; $12: 672-4$

[9] Sasse FJ, "Can we trust end- tidal carbon dioxide measurement in infants?", Journal of Clinical Monitoring 1986; 1:147-148.

[10] Shakya K. and Deegan C, "Determination of the Frequency Response of an End Tidal $\mathrm{CO}_{2}$ analyser", ITB Journal, December 2003; 8:47-56.

[11] Shakya K., Deegan C., Hegarty F. and Markham C., " Measurement of the Frequency Response of Clinical Gas Analysers", ITB Journal, May 2004; 9: 247-257.

[12] Shakya K., Deegan C., Hegarty F. and Markham C., " Design and Implementation of an End Tidal $\mathrm{CO}_{2}$ simulator for the evaluation of Clinical Gas Analysers"; [Accepted, to be published by Rocky Mountain Bioengineering Symposium RMBS2005]

[13] North American Drager, Operators' Instruction manual, 148B Quarry Road, Telford, Pennsylvania 18969

[14] North American Drager, Technical Service Manual, Drager Service, 24 Commerce Drive, Telford, PA 18969

[15] Pneupac, Smiths medical, [Online]. Available: http://www.pneupac.co.uk/Literature/Capnocheck\%20Sleep.pdf

[16] Datex -Ohmeda, Global site, [Online]. Available :

http://www.datexohmeda.com/dl/Modules/8004428.pdf 